\title{
AN EXPLICIT BASIS OF LOWERING OPERATORS FOR IRREDUCIBLE REPRESENTATIONS OF UNITARY GROUPS
}

\author{
D.S. Sage and L. Smolinsky \\ Department of Mathematics, Louisiana State University, Baton Rouge, LA 70803, USA \\ E-mail: sage@math.lsu.edu, smolinsk@math.lsu.edu
}

Received 2 July 2010; revised 13 December 2010; accepted 15 December 2010

\begin{abstract}
The representation theory of the unitary groups is of fundamental significance in many areas of physics and chemistry. In order to label states in a physical system with unitary symmetry, it is necessary to have explicit bases for the irreducible representations. One systematic way of obtaining bases is to generalize the ladder operator approach to the representations of $\mathrm{SU}(2)$ by using the formalism of lowering operators. Here, one identifies a basis for the algebra of all lowering operators and, for each irreducible representation, gives a prescription for choosing a subcollection of lowering operators that yields a basis upon application to the highest weight vector. Bases obtained through lowering operators are particularly convenient for computing matrix coefficients of observables as the calculations reduce to the commutation relations for the standard matrix units. The best known examples of this approach are the extremal projector construction of the Gelfand-Zetlin basis and the crystal (or canonical) bases of Kashiwara and Lusztig. In this paper, we describe another simple method of obtaining bases for the irreducible representations via lowering operators. These bases do not have the algebraic canonicity of the GelfandZetlin and crystal bases, but the combinatorics involved are much more straightforward, making the bases particularly suited for physical applications.
\end{abstract}

Keywords: unitary group, special unitary group, irreducible representations, lowering operators, spin-free quantum chemistry, many-body problem

PACS: 02.20.-a, 31.15.xh

\section{Introduction}

The representation theory of the unitary groups plays a fundamental role in many areas of physics and chemistry. The first and best-known application is the appearance of the special unitary group $\mathrm{SU}(2)$ in the quantum theory of angular momentum [1]. Elliott's $\mathrm{SU}(3)$ model of the nucleus provides a bridge between the standard and collective models [2-4], and various low-dimensional unitary groups have been used in particle physics [5]. More general unitary groups arise in the many-body problem [6, 7], quantum chemistry [8,9], and in quantum computation [10, 11].

To provide some physical insight, we describe the role of the unitary groups in the many-body problem. Consider a system with $n$ single-particle boson or fermion states. The unitary group $\mathrm{U}(n)$ then acts on the corresponding Fock space as well as on the $N$-particle component for each $N$. The unitary symmetry appears naturally from the creation and annihilation operator formalism. If $a_{i}^{*}$ and $a_{i}$ are the creation and annihilation operators for state $i$, let $E_{i j}=a_{i}^{*} a_{j}$, the tran- sition operator from state $j$ to state $i$. These operators satisfy the same commutation relations as the standard basis for the Lie algebra of the $n \times n$ general linear group [12], and accordingly the Lie algebra (i. e., the infinitesimal generators) of $\mathrm{U}(n)$ is spanned by the operators $E_{i j}+E_{j i}$ for $i \leq j$ and i $\left(E_{i j}-E_{j i}\right)$ for $i<j$. (One obtains the usual physics convention for the Lie algebra by multiplying these operators by i.)

A variation of this procedure appears in the spinfree approach to quantum chemistry pioneered by Matsen [9]. In this theory, one studies the electronic structure of molecules by only considering the spatial component of the wavefunctions. Instead of single occupancy fermion orbitals, one considers "freeon" orbitals, which can contain up to two electrons. As Matsen has written, the motivation for this theory is "to separate the spin kinematics (only an indicator) from the freeon dynamics which contains the basic physics, i. e., the spinfree Coulomb repulsion" [13]. In the spin-free unitary group formulation (one of four equivalent formulations of spin-free quantum chemistry [9]), if $n$ is the number 
of freeon orbitals, the Hamiltonian is a quadratic polynomial in the generators of $\mathrm{U}(n)$, and $\mathrm{U}(n)$ acts on the space of freeon wavefunctions.

It is straightforward to give a classification of the irreducible representations for $\mathrm{U}(n)$. All finite-dimensional representations have bases consisting of weight vectors: simultaneous eigenvectors for the $E_{i i} \mathrm{~s}$. The simultaneous eigenvalue will be an $n$-tuple of integers called a weight. In the context of the manybody problem, the $E_{i i}$ s are called number operators, and the eigenvalue of $E_{i i}$ is just the number of particles in state $i$. The collection of weights appearing in an irreducible representation contains a unique maximum (with respect to a certain partial order described in Section 2), which will be a nonincreasing sequence $\left(\lambda_{1}, \ldots, \lambda_{n}\right)$, and the irreducible representations are parameterized by highest weights of this form. For example, in spin-free quantum chemistry, the highest weights corresponding to freeon states will be of the form $(2,2, \ldots, 1,1, \ldots, 0,0, \ldots)$. However, for physical applications, it is not enough to be able to distinguish one irreducible representation from another or even to decompose any given representation into irreducible components; rather, one needs explicit bases for the irreducible representations in order to label states of the physical system and to compute matrix coefficients of observables.

There are several constructions of bases for the irreducible representations. The most classical construction is the Weyl module approach introduced by Schur [14] and popularized by Weyl [15]; here, the irreducible representations are realized as tensors with appropriate symmetry properties. Bases can be given by associating explicit tensors to combinatorial objects called semistandard Young tableaux, which will be defined in Section 2.3.

The basis most commonly used in physical applications is the Gelfand-Zetlin basis. If $\mathrm{U}(n-1)$ is viewed as a subgroup of $\mathrm{U}(n)$ via the obvious embedding into the upper left $n-1 \times n-1$ block, then the irreducible representation $W_{\lambda}$ with highest weight $\lambda$, viewed as a representation of $\mathrm{U}(n-1)$, is simply reducible, i.e., each irreducible component has multiplicity one. More precisely, we have the Weyl Branching Rule [16]:

$$
\left.W_{\lambda}\right|_{\mathrm{U}(n-1)}=\bigoplus_{\mu} W_{\mu}
$$

where the sum runs over all $\mu=\left(\mu_{1}, \ldots, \mu_{n-1}\right)$ satisfying $\lambda_{1} \geq \mu_{1} \geq \lambda_{2} \geq \mu_{2} \geq \cdots \geq \mu_{n-1} \geq \lambda_{n}$. Iterating this procedure using the chain of subgroups $\mathrm{U}(n) \supset \mathrm{U}(n-1) \supset \cdots \supset \mathrm{U}(1)$ gives a decomposi- tion of $W_{\lambda}$ into one-dimensional subspaces, since the irreducible representations of the abelian group $\mathrm{U}(1)$ are one-dimensional. We thereby obtain an orthonormal basis indexed by a highest weight for each $\mathrm{U}(k)$, $1 \leq k \leq n$; explicitly, a state is given by the triangular array of integers

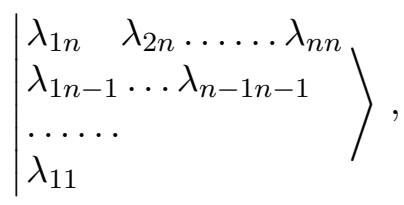

where $\lambda_{i n}=\lambda_{i}$.

It is useful to construct bases of irreducible representations by generalizing the ladder operator approach to the representations of $\mathrm{SU}(2)$ via the formalism of lowering operators. An operator is called a lowering operator if it is a polynomial in the strictly lower triangular matrices $E_{i j}$ for $i>j$. (Sometimes, one allows the diagonal operators $E_{i i}$ as well.) It is well known that every element in $W_{\lambda}$ is obtained by applying a lowering operator to a fixed highest weight vector $v_{\lambda}$. A basis for the irreducible representations can thus be obtained by choosing a collection of lowering operators for each $\lambda$ which upon application to the highest weight vector $v_{\lambda}$ give a basis for $W_{\lambda}$. Since one can assign weights to lowering operators so that the $\mu$ weight space of $W_{\lambda}$ is obtained from $v_{\lambda}$ using operators of weight $\mu-\lambda$, this method can be refined to give bases of each weight space. Such collections of lowering operators give bases independently of any concrete realization of the representations. One can use this approach to compare weight spaces for irreducible representations with different highest weights and even for different $\mathrm{U}(n) \mathrm{s}$ (see Section 3.3). Furthermore, such bases are particularly suited for physical applications because they allow for the mechanical computation of matrix coefficients of observables. Indeed, since most important observables are also polynomials in the $E_{i j}$ s (for any $i, j$ ), matrix coefficients in terms of such a basis may be computed directly from the commutation relations of the $E_{i j} \mathrm{~s}$.

A desirable way to implement the lowering operator method is to start by identifying a basis for the algebra of all lowering operators. One then gives a prescription for choosing for each irreducible representation a subcollection that yields a basis upon application to the highest weight vector.

One scheme for choosing lowering operators gives rise to the Gelfand-Zetlin basis for the irreducible representations. A well-known classical procedure is due to Moshinsky and Nagel [17]. They showed how to find 
explicit lowering operators taking a highest weight vector to the Gelfand-Zetlin states. Effectively, they realized the Gelfand-Zetlin basis through a collection of lowering operators. This could then be used to compute matrix coefficients, as for example in Moshinsky's work on the many-body problem [6].

A more systematic approach to finding these lowering operators uses the theory of extremal projectors discovered by Asherova, Smirnov, and Tolstoy [18, 19] and extensively developed by Zhelobenko [20]. The extremal projector projects a certain extension of the universal enveloping algebra onto its highest weight space along its lower weight spaces. Using the theory of Mickelsson-Zhelobenko algebras [20-23], these operators can be used to obtain the Gelfand-Zetlin bases described above. A more detailed history and development of this approach is given in the survey article [24]. Moreover, extremal projectors lead to Gelfand-Zetlin type bases in a much wider setting. For example, they have been obtained for other classical groups [25], for Lie superalgebras and for quantum groups by Tolstoy [26, 27], and for Yangians by Molev [28]. (There are several other constructions of Gelfand-Zetlin bases. For example, the original construction for quantized enveloping algebras did not use extremal projectors [29, 30].) We also remark that extremal projectors have many other applications in representation theory; see, for example, the review article [31].

Another scheme is delineated in the main theorem of this paper. It gives explicit lowering operators that take highest weight vectors to one of the familiar bases for Weyl modules. The scheme is analogous to Moshinsky and Nagel's work on the Gelfand-Zetlin basis. While these Weyl bases are well-known [32-34], the corresponding lowering operator method is not. One starts with a standard basis of monomial operators for the algebra of lowering operators. There is then a simple combinatorial prescription involving semistandard Young tableaux for selecting those monomial operators that give rise to a basis for a specific irreducible representation.

An additional approach of great current interest are the crystal (or canonical) bases of Kashiwara and Lusztig [35, 36]. Here, the theory of quantum groups is used to construct a basis for the algebra of lowering operators which gives rise to bases for all irreducible representations simultaneously. More specifically, if the distinguished lowering operators are denoted by $P_{i}$, then the set of nonzero elements of the form $P_{i} \cdot v_{\lambda}$ is a basis of $W_{\lambda}$. Unfortunately, this algebraic prescrip- tion for finding the crystal bases is deceptively simple as it is difficult to write down these operators explicitly [37-39].

Both the Moshinsky-Nagel bases and the crystal bases of lowering operators have certain drawbacks. One disadvantage is that the physical significance of the states is somewhat obscure. For instance, in the context of quantum chemistry, Paldus and Sarma have remarked on "the unphysical nature" of the GelfandZetlin basis and have observed that this is a crucial flaw in the valence bond scheme [40]. (See Fig. 1 of [40] for an illustration of this in the case of benzene.) Another is that the lowering operators involved are complicated. Indeed, the Moshinsky-Nagel operators, though explicit, are already unwieldy for SU(4), and no nonalgorithmic formula for the crystal basis operators is known. For physical applications, it would be desirable to have bases of lowering operators which are easier to use. We remark that there is no closed formula for matrix elements in the Weyl basis (as there is for the Gelfand-Zetlin basis). However, for the cases of interest to quantum chemistry, namely representations corresponding to two column Young tableaux, these matrix elements can be computed using algorithmic methods. For example, the Clifford algebra unitary group approach (CAUGA) to quantum chemistry developed by Paldus and Sarma applies to the Weyl basis [40, p. 5137].

The goal of this paper is to find a basis of lowering operators that is as simple as possible. In particular, consider the monomials in the $E_{i j}$ s for $i>j$. The irreducible representation $W_{\lambda}$ is spanned by the monomial lowering operators applied to $v_{\lambda}$. 1 . These states are comparatively easy to interpret physically. For example, in the many-body problem, they are obtained from the highest weight vector by a specific sequence of transitions between single-particle orbitals. It is also easy to compute matrix coefficients in terms of these states. However, in order to determine vectors in an irreducible representation unambiguously, it is still necessary to extract a linearly independent subset, and in physical applications, this has only been done in an $a d$ hoc manner for relatively small cases. We will explain how to overcome this problem.

For example, consider the case of quantum chemistry. The physical intuition provided by the generator state approach leads to it playing a significant role in spin-free quantum chemistry, and Matsen and Pauncz

\footnotetext{
${ }^{1}$ In quantum chemistry, this method is called the generator state approach [41].
} 
devote considerable space in their monograph [9] to the subject. They provide two abstract methods of using the generator states to obtain bases (Gram-Schmidt orthogonalization and a method of Rowe [42] that goes back to Löwdin [43]), but the procedures are not carried out for a general irreducible representations and the resultant basis vectors are not themselves generator states. In fact, Matsen and Pauncz carry out explicit examples only for representations that are small enough to allow one to find bases of generator states by brute force. Our methods produce bases of generator states in general.

In this paper, we will show how to choose explicit monomial lowering operators which give rise to bases for the irreducible representations $W_{\lambda}$ of $\mathrm{U}(n)$, or, more specifically, for its $\mu$ weight space $W_{\lambda}^{\mu}$. Features of this basis include:

1. The bases are obtained by associating monomial lowering operators to certain combinatorial objects called semistandard Young tableaux. Accordingly, they are easily calculated by computer.

2. The lowering operators are left unchanged if the same multiple of $(1, \ldots, 1)$ is added to both $\lambda$ and $\mu$.

3. The lowering operators are independent of $n$ in a sense made precise in Corollary 18.

4. The lowering operators for the weight space $W_{\lambda}^{\mu}$ depend only on the difference $\lambda-\mu$ for $\lambda$ generic (in a sense given in Corollary 19p.

Subsequent to proving Theorem 3, the authors became aware of the precedent of Carter and Lusztig [44]. This paper is a deep and technical paper in which the classical theory of polynomial representations of $\mathrm{GL}_{n}(\mathbf{C})$ is extended to the modular (i. e. positive characteristic) theory using tools such as the Kostant $\mathbf{Z}$-form of the universal enveloping algebra and the affine Weyl group, and it includes a version of Theorem 3 [44, Section 3.5]. Nevertheless, the present paper contains a new proof requiring only textbook Lie theory, and the result does not seem to be well known in the physics and general mathematics communities. We have also demonstrated some properties of these bases that were not given in [44].

We conclude the introduction with a brief description of the rest of the paper. In Sec. 2, we recall some basic facts about the representation theory of the unitary groups. In the following section, we show how to associate monomial lowering operators to semistandard Young tableaux and state our main theorem. We also provide some examples. In particular, we discuss the eightfold way (or equivalently, the three freeon orbital, three electron problem) and the 56-dimensional representation of $\mathrm{SU}(6)$ that describes low-lying baryons. Next, we give some properties of the bases. Finally, we prove the main result in Sec. 4 .

\section{Preliminaries}

We will restrict attention for the present to $\mathrm{SU}(n)$ and discuss the necessary modifications to extend our results to $\mathrm{U}(n)$ later. It will be convenient to work with the Lie algebra $\mathfrak{s l}_{n}(\mathbf{C})$, which has the same finitedimensional representations as $\mathrm{SU}(n)$.

\subsection{Irreducible representations of $\mathfrak{s l}_{n}(\mathbf{C})$}

We begin by recalling the basics of the representation theory of $\mathfrak{s l}_{n}(\mathbf{C})$ (see for example [34]). Let $\mathfrak{h}$ be the subalgebra of $\mathfrak{s l}_{n}(\mathbf{C})$ consisting of the diagonal tracefree matrices; it is called a Cartan subalgebra. A weight vector $v$ for a representation $V$ of $\mathfrak{s l}_{n}(\mathbf{C})$ is a simultaneous eigenvector for the action of $\mathfrak{h}$. The simultaneous eigenvalue will be a linear functional $\lambda$ on $\mathfrak{h}$ called a weight; we let $V^{\lambda}$ denote the corresponding weight space. An element of $\mathfrak{h}^{*}$, the dual space of $\mathfrak{h}$, is determined by the $n-1$-tuple consisting of its values on any basis of $\mathfrak{h}$, typically by its values on the diagonal matrices $H_{i}=E_{i i}-E_{i+1, i+1}$ for $1 \leq i \leq n-1$. (These $H_{i} \mathrm{~s}$ are called simple coroots.) However, it will be more convenient to describe $\mathfrak{h}^{*}$ in terms of the dual space of the algebra $\mathfrak{d}$ of diagonal matrices (with no restriction on the trace). Let $\left\{L_{i}\right\}$ be the dual basis to the basis $\left\{E_{i i}\right\}$ for $\mathfrak{d}$, so $L_{i}\left(E_{j j}\right)=\delta_{i j}$. An element of $\mathfrak{d}^{*}$ can be written uniquely as $\sum_{i=1}^{n} a_{i} L_{i}$, which we will often view as the $n$-tuple $\left(a_{1}, \ldots, a_{n}\right)$. Each $L_{i}$ restricts to give a functional on $\mathfrak{h}$, but of course they are no longer independent. Viewed as elements in $\mathfrak{h}^{*}$, they are subject to the condition $\operatorname{tr}=\sum_{i=1}^{n} L_{i}=0$. Thus, $\lambda \in \mathfrak{h}^{*}$ can be viewed an $n$-tuple, well-defined up to the addition of a real number $a$ to each coordinate. This means that formally we can express $\mathfrak{h}^{*}$ as a quotient space:

$$
\mathfrak{h}^{*}=\left\{\sum_{i=1}^{n} a_{i} L_{i} \mid a_{i} \in \mathbf{C}\right\} /\left\{a \sum_{i=1}^{n} L_{i} \mid a \in \mathbf{C}\right\} .
$$

We get a unique representative for a functional by normalizing so that $a_{n}=0$.

Every finite-dimensional representation of $\mathfrak{s l}_{n}(\mathbf{C})$ is a sum of weight spaces, and those weights appearing in 
this way can be expressed as $n$-tuples of integers. Accordingly, the possible weights form the weight lattice:

$$
\Lambda_{W}=\left\{\sum_{i=1}^{n} \lambda_{i} L_{i} \mid \lambda_{i} \in \mathbf{Z}\right\} /\left\{a \sum_{i=1}^{n} L_{i} \mid a \in \mathbf{Z}\right\} .
$$

Again, we can normalize the coordinates for a weight so that $\lambda_{n}=0$. However, we will also have occasion to use another normalization. Note that $\sum_{i=1}^{n} \lambda_{i}$ is welldefined modulo $n$, so if $d \in \mathbf{Z}$ is congruent to this sum, we can find a representative for the weight whose coordinates sum to $d$.

The nonzero weights of the adjoint representation (i. e., $\mathfrak{s l}_{n}(\mathbf{C})$ acting on itself via $x \cdot y=x y-y x$ ) are called roots. The roots of $\mathfrak{s l}_{n}(\mathbf{C})$ are $L_{i}-L_{j}$ for $i \neq j$ with corresponding root vector $E_{i j}$. Recall that the standard choice of simple positive roots for $\mathfrak{s l}_{n}(\mathbf{C})$ is $\alpha_{i}=L_{i}-L_{i+1}$ for $1 \leq i \leq n-1$. With this choice, the root vector $E_{i j}$ is positive (resp. negative) if $i<j$ (resp. $i>j$ ). The span of the positive root vectors forms the subalgebra $\mathfrak{n}$ of strictly upper triangular matrices; similarly, the negative root vectors span the subalgebra $\overline{\mathfrak{n}}$ of strictly lower triangular matrices. The weight lattice admits a partial order defined by $\mu \prec \lambda$ if and only if $\lambda-\mu$ is a nonnegative integral linear combination of the $\alpha_{i}$ s. It is immediate that the action of positive (resp. negative) roots takes a weight space to a weight space with a higher (resp. lower) weight.

A weight $\lambda$ is called dominant if $\lambda\left(H_{i}\right) \geq 0$ for $1 \leq i \leq n-1$. Concretely, $\lambda=\lambda_{1} L_{1}+\ldots \lambda_{n-1} L_{n-1}$ is dominant if and only if $\lambda_{1} \geq \lambda_{2} \geq \cdots \geq \lambda_{n-1} \geq$ 0 . There is a one-to-one correspondence between irreducible representations and dominant weights. If $V_{\lambda}$ is the irreducible representation corresponding to the dominant weight $\lambda$, then $\lambda$ is the highest weight in $V_{\lambda}$ with respect to the partial order given above, and the highest weight vector is unique up to scalar.

\subsection{Raising and lowering operators}

We now describe the algebras of raising and lowering operators. The universal enveloping algebra $U\left(\mathfrak{s l}_{n}(\mathbf{C})\right)$ is the associative algebra generated by the elements of $\mathfrak{s l}_{n}(\mathbf{C})$ with commutation relations determined by the Lie bracket of $\mathfrak{s l}_{n}(\mathbf{C})$. The universal enveloping algebra has the property that any representation of $\mathfrak{s l}_{n}(\mathbf{C})$ is also a representation of $\mathcal{U}\left(\mathfrak{s l}_{n}(\mathbf{C})\right)$ and vice versa. Given an ordered basis $X_{1}, \ldots, X_{n^{2}-1}$ for $\mathfrak{s l}_{n}(\mathbf{C})$, the elements of $\mathcal{U}\left(\mathfrak{s l}_{n}(\mathbf{C})\right)$ are polynomials in the $X_{i} \mathrm{~s}$, and by the Poincaré-Birkhoff-Witt theorem, the monomials $X_{1}^{i_{1}} \ldots X_{n^{2}-1}^{i_{n^{2}-1}}$ are a basis for $\mathcal{U}\left(\mathfrak{s l}_{n}(\mathbf{C})\right)$. We will always use the basis for $\mathfrak{s l}_{n}(\mathbf{C})$ consisting of the sim- ple root vectors $E_{i j}$ and simple coroots $H_{i}$. We will not specify the order now, but it will respect the order in the direct sum decomposition $\mathfrak{s l}_{n}(\mathbf{C})=\overline{\mathfrak{n}} \oplus \mathfrak{h} \oplus \mathfrak{n}$, , so that the lower triangular matrices come first, the diagonal matrices second, and the upper triangular matrices last. The algebra of raising operators for $\mathfrak{s l}_{n}(\mathbf{C})$ is the subalgebra consisting of polynomials in the positive root vectors; it is isomorphic to $U(\mathfrak{n})$, the universal enveloping algebra for $\mathfrak{n}$. Similarly, the algebra of lowering operators is just $\mathcal{U}(\overline{\mathfrak{n}})$, which may be viewed as the subalgebra of polynomials in the negative root vectors.

It is well known that if $V$ is irreducible with highest weight vector $v$, then $V=\mathcal{U}(\overline{\mathfrak{n}}) \cdot v$, so that a spanning set for $V$ is obtained by applying all monomial lowering operators to $v$. We will abuse terminology slightly and say that $V$ is spanned by lowering operators. (In fact, it is possible to realize $V$ as a quotient of $\mathcal{U}\left(\mathfrak{s l}_{n}(\mathbf{C})\right)$ by a certain left ideal in such a way that the coset of 1 is a highest weight vector [45]. This means that $V$ is spanned by the images of lowering operators in this quotient space.) Note that the lowering operator $\prod_{i>j} E_{i j}^{k_{i j}}$ (with the product taken in some fixed order) sends the weight space $V^{\lambda}$ to $V^{\mu}$, where

$$
\mu_{i}=\lambda_{i}+\sum_{j<i} k_{i j}-\sum_{j>i} k_{j i} .
$$

This implies that $\sum \mu_{i}=\sum \lambda_{i}$. These sums are actually only well-defined modulo $n$, so we obtain the fact:

If the weight space $V_{\lambda}^{\mu} \neq 0$, then $\sum \mu_{i} \equiv \sum \lambda_{i}$ $\bmod n$.

We now normalize the weights appearing in $V_{\lambda}$ as follows:

1. The dominant weight $\lambda$ is chosen with $\lambda_{n}=0$.

2. All other weights are chosen so that their coordinates sum to $\sum \lambda_{i}$.

Monomial lowering operators are also weight vectors; the corresponding weights may be normalized so that their coordinates sum to zero. With these normalizations, the monomial lowering operator given above taking $V^{\lambda}$ to $V^{\mu}$ has weight $\mu-\lambda$.

It is clear that the lowering operators for $\mathfrak{s l}_{n}(\mathbf{C})$ are also lowering operators for $\mathfrak{s l}_{m}(\mathbf{C})$ if $m \geq n$. Accordingly, it will be convenient to have a universal algebra Low containing all lowering operators independent of $n$. This algebra is generated by all $E_{i j}$ with $i>j \geq 1$ subject to the obvious commutation re-

\footnotetext{
${ }^{2}$ We denote these algebras by $\overline{\mathfrak{n}}_{n}, \mathfrak{h}_{n}$, and $\mathfrak{n}_{n}$, if the ambient $\mathfrak{s l}_{n}(\mathbf{C})$ is not clear from context.
} 
lations. More formally, let $\mathfrak{s l}_{\infty}(\mathbf{C})=\bigcup_{n>1} \mathfrak{s l}_{n}(\mathbf{C})$; it is the Lie algebra of infinite square matrices (with entries indexed by ordered pairs of positive integers) with only finitely many nonzero entries and whose diagonal entries sum to 0 . The lower triangular subalgebra $\overline{\mathfrak{n}}_{\infty}$ is also an increasing union of this form, and Low $=\mathcal{U}\left(\overline{\mathfrak{n}}_{\infty}\right)=\bigcup_{n \geq 1} U\left(\overline{\mathfrak{n}}_{n}\right) \subset \mathcal{U}\left(\mathfrak{s l}_{\infty}\right)$.

\subsection{Semistandard Young tableaux}

In order to describe an explicit basis of monomial lowering operators, we will need to introduce some combinatorial machinery. If $d$ is a positive integer, a partition $\lambda$ of $d$ is a nonincreasing sequence $\left(\lambda_{1}, \ldots, \lambda_{k}\right)$ of positive integers which sum to $d$. The Young diagram associated to $\lambda$ is a left-justified collection of boxes, with $\lambda_{i}$ boxes in row $i ; \lambda$ is called the shape of the diagram. $\mathrm{B}$ A Young tableau is obtained by filling in the boxes of a Young diagram with positive integers. The shape of a tableau $T$ is denoted by $\operatorname{sh}(T)$. We say that a Young tableau with $d$ boxes is semistandard if the numbers assigned to the boxes are integers from 1 to $d$ with the entries in each row nondecreasing and the entries in each column increasing. If $\mu$ is another partition of $d$ (or any sequence of nonnegative integers summing to $d$ ), then a tableau is called semistandard on $\lambda$ of content $\mu$ if it is semistandard with shape $\lambda$ and contains $\mu_{1} 1 \mathrm{~s}, \mu_{2} 2 \mathrm{~s}$, etc. We denote the content of $T$ by $\operatorname{co}(T)$. A sequence $\mu$ which appears as the content of a semistandard tableau with shape $\lambda$ is called an admissible content for $\lambda$. The number of tableaux with shape $\lambda$ and content $\mu$ is the Kostka number $K_{\lambda \mu}$. We will denote the set of such semistandard tableaux by $\mathcal{S}_{\lambda}^{\mu}$ and the set of all semistandard tableaux on $\lambda$ by $\mathcal{S}_{\lambda}=\bigcup_{\mu} \mathcal{S}_{\lambda}^{\mu}$. The analogous set of tableaux where entries are restricted to $\{1, \ldots n\}$ (equivalently, $\mu$ has length at most $n$ ) will be denoted by $\mathcal{S}_{\lambda}(n)$. Finally, $\mathcal{S}(n)$ will denote the set of all semistandard tableaux with $\lambda$ (resp. $\mu$ ) of length less than (resp. at most) $n$, and $\mathcal{S}$ will denote the set of all semistandard tableaux.

It is clear that the dominant weights for $\mathfrak{s l}_{n}(\mathbf{C})$ correspond to Young diagrams with at most $n-1$ rows. We use $\lambda$ to denote both the dominant weight $\lambda_{1} L_{1}+$ $\cdots+\lambda_{n-1} L_{n-1}$ and the partition $\left(\lambda_{1}, \ldots, \lambda_{n-1}\right)$. It is less obvious that a weight $\mu$ appearing in $V_{\lambda}$ can be interpreted as the content of a semistandard tableau on $\lambda$. To see this, one must show that each $\mu_{i}$ is nonneg-

\footnotetext{
${ }^{3}$ It will sometimes be convenient to allow partition sequences to end with a string of zeros. Of course, sequences determine the same partition and Young diagram when their nonzero entries coincide.
}

ative and moreover that $\sum_{i=1}^{s} \mu_{i} \leq \sum_{i=1}^{s} \lambda_{i}$ for all $s$ (because in a semistandard tableau, an integer $\leq s$ can only appear in the first $s$ rows). The second statement follows immediately from equation (2), since this equation gives $\sum_{i=1}^{s} \mu_{i}=\sum_{i=1}^{s} \lambda_{i}-\sum_{i=1}^{s} \sum_{j>s} k_{j i}$. To prove that $\mu_{i} \geq 0$, it suffices to show that $\mu_{1} \geq 0$, since the set of weights in $V_{\lambda}$ is closed under permutations [34]. The lowest weight appearing in $V_{\lambda}$ is $\nu=\left(0, \lambda_{n-1}, \ldots, \lambda_{1}\right)$, and $V_{\lambda}^{\mu}$ is generated by applying appropriate monomial raising operators $\prod_{i<j} E_{i j}^{k_{i j}}$ to a lowest weight vector. In particular, one obtains $\mu_{1}=\sum_{j>1} k_{1 j} \geq 0$. Thus, $\mu$ makes sense as the content of a tableau. In fact, it is known that $\operatorname{dim} V_{\lambda}^{\mu}=$ $K_{\lambda \mu}$ [34].

\section{Bases of monomial lowering operators}

\subsection{Semistandard tableaux and lowering operators}

We are now ready to define the lowering operators that will provide bases for the irreducible representations of the unitary groups. In particular, we show how to associate a monomial lowering operator to any semistandard tableau.

Consider Hebrew lexicographic order on the set $\{(s, i) \mid s>i \geq 1\}$, i.e., $(s, i)<(t, j)$ if $i<j$ or $i=j$ and $s<t$. The corresponding order on the $E_{s i} \mathrm{~s}$ induces a Poincaré-Birkhoff-Witt basis of Low and each $\mathcal{U}(\overline{\mathfrak{n}})$.

We now assign an element of this basis to any semistandard tableau. Let $T$ be a semistandard tableau. Note that $T_{i j} \geq i$. For each $s>i$, let $a_{s i}=\#\left\{j \mid T_{i j}=\right.$ $s\}$, the number of times $s$ appears in row $i$. We define a map $\gamma: \mathcal{S} \rightarrow$ Low by setting $\gamma(T)$ to be the monomial lowering operator consisting of the $E_{s i}^{a_{s i}}$ s written in the order described above:

$$
\gamma(T)=E_{21}^{a_{21}} \ldots E_{n 1}^{a_{n 1}} E_{32}^{a_{32}} \ldots E_{n n-1}^{a_{n n-1}} .
$$

In other words, $\gamma(T)$ is obtained by sweeping through $T$ from left to right and top to bottom and writing down the negative root vector $E_{s i}$ each time one reaches a box in the $i$ th row containing the entry $s>i$.

Remark 1. Equation (3) defining $\gamma$ makes sense for general tableaux $T$ with $T_{i j} \geq i$. Furthermore $\gamma(S)=$ $\gamma(T)$ if the content of each row is the same for $S$ and $T$. 
Example 2.

$T=$\begin{tabular}{l|l|l|l|l|l|}
\hline & 2 & 3 & 3 & 5 \\
\hline 2 & 4 & 4 & & & \\
\cline { 1 - 2 } 3 & 5 & & & &
\end{tabular}$(T)=E_{21} E_{31}^{2} E_{51} E_{42}^{2} E_{53} E_{54}$.

Of course, $\gamma(T)$ will only be a lowering operator for $\mathfrak{s l}_{n}(\mathbf{C})$ if $T$ has at most $n-1$ rows and no entry is greater than $n$. Thus, if the length of $\lambda$ is smaller than $n, \gamma$ restricts to give a function $\gamma_{n \lambda}: \mathcal{S}_{\lambda}(n) \rightarrow \mathcal{U}\left(\overline{\mathfrak{n}}_{n}\right)$. Moreover, $\gamma_{n \lambda}$ restricts to define maps $\gamma_{\lambda}^{\mu}: \mathcal{S}_{\lambda}^{\mu} \rightarrow \mathcal{U}\left(\overline{\mathfrak{n}}_{n}\right)^{\mu-\lambda}$ for each admissible $\mu$ of length at most $n$.

As we will see in Sec. 3.3, the image of $\gamma$ is the entire given PBW basis of Low while $\gamma(\mathcal{S}(n))$ is the PBW basis for $\mathcal{U}\left(\overline{\mathfrak{n}}_{n}\right)$. In fact, it is possible to choose a minimal tableau representing any basis element.

\subsection{Main theorem and examples}

We are now ready to state the main theorem.

Theorem 3. Let $V_{\lambda}$ be the irreducible representation of $\mathrm{SU}(n)$ with highest weight $\lambda$ and highest weight vector $v_{\lambda}$. Then if $\mu$ is any admissible content of length at most $n$, the set $\left\{\gamma_{\lambda}^{\mu}(T) \cdot v_{\lambda} \mid T \in \mathcal{S}_{\lambda}^{\mu}\right\}$ is a basis for the weight space $V_{\lambda}^{\mu}$. In particular, $\left\{\gamma_{n \lambda}(T) \cdot v_{\lambda} \mid T \in \mathcal{S}_{\lambda}(n)\right\}$ is a basis for $V_{\lambda}$.

It should be noted that if $\mu$ is an admissible content for $\lambda$ which is not a weight appearing in $\lambda$, then $\gamma_{\lambda}^{\mu}(T) \cdot v_{\lambda}=0$ for every semistandard $T$ with this content. However, it is not true that if $T \notin \mathcal{S}_{\lambda}(n)$, then $\gamma(T) \cdot v_{\lambda}=0$. This is unlike the situation for crystal bases, where the basis operators giving the basis for $V_{\lambda}$ are precisely those which do not kill $v_{\lambda}$.

The proof of the theorem will be given in Section 4 .

Remark 4. There are orders besides the Hebrew lexicographic order that yield an analogue of Theorem 3, but with different bases for the irreducible representations.

Remark 5. It is of course possible to give a version of this theorem (and of all other results in this paper) in terms of raising operators.

Example 6. For $\mathrm{SU}(2)$, the partition $\lambda$ is just a nonnegative integer $p$, so that the Young diagram has a single row. The admissible contents with length at most 2 are $(i, p-i)$ for $0 \leq i \leq p$, giving rise to tableaux of the form $\sqrt{[1|1| 2|2| 2 \mid}$. The corresponding basis of lowering operators for $V_{p}$ is $\left\{E_{21}^{i} \mid 0 \leq i \leq p\right\}$. Up to normalization, these are just the usual lowering operators $J_{-}^{i}$ on the irreducible representation with total angular momentum $p / 2$ [1].

The next two examples come from particle physics (and quantum chemistry).

Example 7. (The eightfold way) The baryon and meson octets are described by the adjoint representation of SU(3) with Young diagram $\square[5]$. The basis elements obtained by applying the lowering operators from Theorem 3 to the highest weight vector $v_{\lambda}=E_{13}$ are given in Table 1 as are the corresponding baryon and meson states. The weights are also given in terms of the charge $Q$ and strangeness $S$ quantum numbers.

The basis given in the table can also be interpreted as a basis of generator states in spin-free quantum chemistry. Indeed, the irreducible representation of $\mathrm{U}(3)$ with highest weight $(2,1,0)$ corresponds to the doublet space for a system with three freeon orbitals and three electrons (e. g., the allyl radical); concretely, it consists of states with two electrons in one orbital and a single electron in another. The basis is obtained by applying the $\gamma_{\lambda}^{\mu}(T)$ to the highest weight state with two electrons in the lowest state and the third in the middle state. Matsen works out this example in detail in [46].

Example 8. The low-lying baryons are described by the 56-dimensional representation of SU(6) with Young

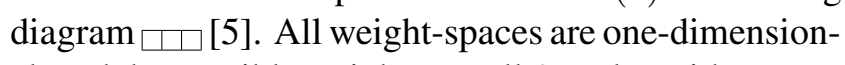
al, and the possible weights are all 6-tuples with nonvanishing entries $\{3\},\{2,1\}$, and $\{1,1,1\}$. If the first entry of the weight vector is nonzero, then the corresponding lowering operators are $I, E_{i 1}, E_{i 1}^{2}$, and $E_{i 1} E_{j 1}$; otherwise, the operators are $E_{i 1}^{3}, E_{i 1}^{2} E_{j 1}, E_{i 1} E_{j 1}^{2}$, and $E_{i 1} E_{j 1} E_{k 1}$. Here, $2 \leq i<j<k$.

Example 9. Let $\lambda=4 L_{1}+2 L_{2}+L_{3}$ and $\mu=2 L_{1}+$ $2 L_{2}+2 L_{3}+L_{4}$. For any $\mathrm{SU}(n)$ with $n \geq 4$, the weight space $V_{\lambda}^{\mu}$ is 6-dimensional. The semistandard tableaux and corresponding lowering operators are given in Table 2. The third column of the table is explained in Example 14 .

\subsection{Properties of the bases}

It is evident from the construction that different semistandard tableaux can give rise to the same lowering operator; we call such tableaux operator equivalent. The tableaux $S$ and $T$ are operator equivalent if $\gamma(S)=$ 
Table 1. The baryon and meson octets.

\begin{tabular}{|c|c|c|c|c|c|c|}
\hline$\mu$ & $(Q, S)$ & $T$ & $\gamma_{\lambda}^{\mu}(T)$ & $\gamma_{\lambda}^{\mu}(T) \cdot v_{\lambda}$ & baryon & meson \\
\hline$(2,1,0)$ & $(1,0)$ & $\frac{11}{2}{ }^{1}$ & $I$ & $E_{13}$ & $p$ & $K^{+}$ \\
\hline$(2,0,1)$ & $(1,-1)$ & $\left.\frac{11}{3}\right]^{1}$ & $E_{32}$ & $-E_{12}$ & $-\Sigma^{+}$ & $-\pi^{+}$ \\
\hline$(1,2,0)$ & $(0,0)$ & $\frac{12}{2}$ & $E_{21}$ & $E_{23}$ & $n$ & $K^{0}$ \\
\hline \multirow[t]{2}{*}{$(1,1,1)$} & $(0,-1)$ & $\frac{12}{3}$ & $E_{21} E_{32}$ & $E_{11}-E_{22}$ & $\sqrt{2} \Sigma^{0}$ & $\sqrt{2} \pi^{0}$ \\
\hline & & $\frac{12^{3}}{2}$ & $E_{31}$ & $E_{33}-E_{11}$ & $-\frac{1}{\sqrt{2}} \Sigma^{0}+\sqrt{\frac{3}{2}} \Lambda$ & $-\frac{1}{\sqrt{2}} \pi^{0}+\sqrt{\frac{3}{2}} \eta$ \\
\hline$(1,0,2)$ & $(0,-2)$ & $\left.\frac{13}{3}\right]^{3}$ & $E_{31} E_{32}$ & $-E_{32}$ & $-\Xi^{0}$ & $-\bar{K}^{0}$ \\
\hline$(0,2,1)$ & $(-1,-1)$ & $\left.\frac{22}{3}\right]^{2}$ & $E_{21}^{2} E_{32}$ & $2 E_{21}$ & $2 \Sigma^{-}$ & $2 \pi^{-}$ \\
\hline$(0,1,2)$ & $(-1,-2)$ & $\frac{22^{3}}{3}$ & $E_{21} E_{31} E_{32}$ & $E_{31}$ & $\Xi^{-}$ & $K^{-}$ \\
\hline
\end{tabular}

Table 2. Lowering operators for $\lambda=4 L_{1}+2 L_{2}+L_{3}$ and $\mu=2 L_{1}+2 L_{2}+2 L_{3}+L_{4}$.

\begin{tabular}{|c|c|c|}
\hline$T$ & $\gamma_{\lambda}^{\mu}(T)$ & minimum equivalent tableau \\
\hline \begin{tabular}{|l|l|l|}
1 & 1 & 2 \\
3 & 3 & 2 \\
4 & & \\
\end{tabular} & $E_{21}^{2} E_{32}^{2} E_{43}$ & \begin{tabular}{|l|l|}
$2|2|$ \\
3 & 3 \\
4 & \\
\end{tabular} \\
\hline \begin{tabular}{|l|l|l|}
$\frac{1}{2}$ & $|2| 3 \mid$ \\
4 & 3 & \\
\end{tabular} & $E_{21} E_{31} E_{32} E_{43}$ & \begin{tabular}{|l|}
$\frac{2}{3}$ \\
$\frac{3}{4}$ \\
\end{tabular} \\
\hline 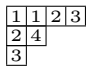 & $E_{21} E_{31} E_{42}$ & $\frac{23^{3}}{4}$ \\
\hline 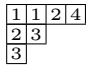 & $E_{21} E_{41} E_{32}$ & $\begin{array}{l}\frac{24}{3} \\
\end{array}$ \\
\hline \begin{tabular}{|l|l|l|l|}
1 & 1 & 3 & 3 \\
2 & 2 & \\
4 & & \\
\end{tabular} & $E_{31}^{2} E_{43}$ & \begin{tabular}{|l|l|l|}
1 & 3 & 3 \\
2 & & \\
4 & &
\end{tabular} \\
\hline 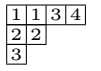 & $E_{31} E_{41}$ & $3 \mid 4$ \\
\hline
\end{tabular}

$\gamma(T)$. More explicitly, $S$ and $T$ are operator equivalent if they have the same number of entries $s$ in row $j$ for each $j$ and $s>j$.

Lemma 10. Any operator in the PBW basis for $\mathcal{U}\left(\overline{\mathfrak{n}}_{n}\right)$ is realized by a tableau in $\mathcal{S}(n)$.

Proof Consider a basis operator $A=\prod_{j<i \leq n} E_{i j}^{a_{i j}}$. We produce a semistandard tableau $S_{A}$ of shape $\lambda$, where $\lambda=\left(\lambda_{1}, \ldots, \lambda_{n-1}\right)$, with $\lambda_{i}$ defined as follows. Let $\lambda_{n}=0$ and $\lambda_{j}=\lambda_{j+1}+\sum_{i>j} a_{i j}$. The $j$ th row of $S_{A}$ will contain $\lambda_{j+1}$ entries $j$ and $a_{i j}$ entries $i$ for $i>j$. The resulting tableau is semistandard since all entries in row $j+1$ are at least $j+1$, and if an entry in the $j$ th row is above a box, then its entry is $j$.

Corollary 11. There is a one-to-one correspondence between the PBW basis for $\mathcal{U}\left(\overline{\mathfrak{n}}_{n}\right)$ (resp. Low) and operator equivalence classes of tableaux in $\mathcal{S}(n)$ (resp. $\mathcal{S}$ ).
We remark that once $\lambda$ and $\mu$ are fixed, there is at most one element of $\mathcal{S}_{\lambda}^{\mu}$ in each equivalence class.

The representative of each operator equivalence class constructed in the proof of the lemma is usually considerably bigger than necessary. In fact, there is a smallest representative of each class. Given two nonincreasing sequences $\lambda$ and $\lambda^{\prime}$, we say that $\lambda \ll \lambda^{\prime}$ if $\lambda^{\prime}-\lambda$ is a nonincreasing, nonnegative sequence.

\section{Proposition 12.}

1. Given $A$ an operator in the PBW basis for Low, there is a unique semistandard tableau $T_{A}$ such that $\gamma\left(T_{A}\right)=A$ and, for any $S \in \mathcal{S}$ with $\gamma(S)=A$, $\operatorname{sh}(S) \gg \operatorname{sh}\left(T_{A}\right)$. If $A \in \mathcal{U}\left(\overline{\mathfrak{n}}_{n}\right)$, then $T_{A} \in \mathcal{S}(n)$.

2. Suppose that $A$ has weight $\tau$. Then there exists $S \in$ $\mathcal{S}_{\lambda}^{\mu}$ such that $\gamma(S)=A$ if and only if $\tau=\mu-\lambda$, $\operatorname{sh}(S) \gg \operatorname{sh}\left(T_{A}\right)$, and $\operatorname{co}(S) \gg \operatorname{co}\left(T_{A}\right)$. In this case, $\operatorname{sh}(S)-\operatorname{sh}\left(T_{A}\right)=\operatorname{co}(S)-\operatorname{co}\left(T_{A}\right)$, and $S$ is obtained from $T_{A}$ by adding $b_{k}$ boxes with entry $k$ to row $k$, where $b_{i}=\left(\operatorname{sh}(S)-\operatorname{sh}\left(T_{A}\right)\right)_{i}$.

Example 13. The tableau in Example 2 is the smallest representative of its operator equivalence class.

Example 14. None of the tableaux in Table 2 are minimum representatives. The smallest representative in each operator class is given in the third column of the table.

Remark 15. An operator $A$ may be represented by a tableau that is smaller than any representation as a semistandard tableau (cf. Remark 1); indeed, $A$ may be represented by a tableau of shape $\lambda$ if and only if $\sum_{i>j} a_{i j} \leq \lambda_{j}$ for each $j$. If $A$ cannot be represented as a possibly non-semistandard tableau of shape $\lambda$, i. e., 
$A$ does not fit inside $\lambda$, then $A \cdot v_{\lambda}=0$. This is shown in Proposition 24.

Proof Fix a basis operator $A \in \mathcal{U}\left(\overline{\mathfrak{n}}_{n}\right)$. The tableau $T_{A}$ is constructed recursively from the tableau $S_{A}$ given in the proof of Lemma 10. The shape of $T_{A}$ has length $n-1$, and its last row agrees with the last row of $S_{A}$. Assume that the $j$ th row of $T_{A}$ has been constructed $(2 \leq j \leq n-1)$. Remove the boxes in row $j-1$ of $S_{A}$ containing $j-1$ s and left justify the remaining boxes. Slide these boxes the minimum number of spaces to the right so that all entries are strictly smaller than those in the $j$ th row of $T_{A}$, and add new boxes containing $j-1$ s to fill out the row to the left. This is row $j-1$ of $T_{A}$. Let $\lambda^{A}=\operatorname{sh}\left(T_{A}\right)$ and $\mu^{A}=\operatorname{co}\left(T_{A}\right)$. Note that $\tau=\mu^{A}-\lambda^{A}$.

Now suppose $S \in \mathcal{S}_{\lambda}^{\mu}$ and $\gamma(S)=A$. It is trivial that $\tau=\mu-\lambda$. Let $b=\operatorname{sh}(S)-\lambda^{A}$. We will show by downward induction that $b$ is a nonincreasing sequence of nonnegative integers and that the last $\lambda_{j}^{A}$ entries of $S_{j}$ (i. e., the $j$ th row of $S$ ) coincide with $\left(T_{A}\right)_{j}$. We first note that if $j \geq n$, then $S_{j}$ contains $b_{j} j$ s and no other entries. This implies that $b_{j} \geq 0$; moreover, since $S$ is a tableau, $b_{n} \geq b_{n+1} \geq b_{n+2} \geq \ldots$. Next, each box in $S_{n}$ must be underneath an $n-1$. Since $S_{n-1}$ contains exactly $b_{n-1} n-1 \mathrm{~s}$, we obtain $b_{n-1} \geq b_{n}$. Also, $\left(T_{A}\right)_{n-1}$ contains no $n-1$ s, so the last $\lambda_{n-1}^{A}$ entries of $S_{n-1}$ are just $\left(T_{A}\right)_{n-1}$.

Finally, assume that $b_{j}<b_{j+1}$ with $j \leq n-2$. Consider the semistandard tableau with two rows obtained by omitting the first $\left|S_{j+1}\right|-\lambda_{j+1}^{A}$ boxes from $S_{j}$ and $S_{j+1}$. By inductive hypothesis, the lower row is $\left(T_{A}\right)_{j+1}$. The entries greater than $j$ in the upper row are the same as those in $\left(T_{A}\right)_{j}$, but it has strictly fewer entries equal to $j$. Since every entry in the upper row is larger than the entry below, this contradicts the definition of $\left(T_{A}\right)_{j}$. Hence, $b_{j} \geq b_{j+1}$. Since $\gamma(S)=\gamma\left(T_{A}\right)$, the last $\lambda_{j}^{A}$ entries of $S_{j}$ are $\left(T_{A}\right)_{j}$. We have thus shown that $b$ is a nonnegative, nonincreasing sequence and that $\operatorname{co}(S)-\operatorname{co}\left(T_{A}\right)=b$.

For the converse, assume that $\lambda \gg \operatorname{sh}\left(T_{A}\right)$ and $\mu \gg$ $\operatorname{co}\left(T_{A}\right)$ with $\mu-\lambda=\tau$. Let $S$ be the tableau of shape $\lambda$ obtained by adjoining $b_{j}=\left(\lambda-\lambda^{A}\right)_{j}$ boxes with entry $j$ to the left of each row of $T_{A}$. We see immediately that $\gamma(S)=A$ and $\mu(S)=\mu^{A}+\left(\lambda-\lambda^{A}\right)=\lambda+$ $\mu-\lambda=\mu$, so it only remains to check that $S$ is semistandard. Let $(j, k)$ be the coordinates of a box that is above another box. If $k \leq b_{j}$, then $S_{j+1, k} \geq j+1>$ $j=S_{j k}$. Otherwise, $k>b_{j} \geq b_{j+1}$, so $S_{j+1, k}=$ $\left(T_{A}\right)_{j+1, k-b_{j+1}}>\left(T_{A}\right)_{j, k-b_{j+1}} \geq\left(T_{A}\right)_{j, k-b_{j}}=S_{j k}$ as desired. This concludes the proof.
We can now determine when the bases for $V_{\lambda}^{\mu}$ and $V_{\lambda^{\prime}}^{\mu^{\prime}}$ given in Theorem 3 come from the same set of lowering operators. We denote the (finite) PBW basis of Low ${ }^{\mu}$ by $\left\{A_{q}^{\mu}\right\}$, where $q$ varies over an index set.

Theorem 16. Let $V_{\lambda}$ be the irreducible representation of $\mathrm{SU}(n)$ with highest weight $\lambda$ and highest weight vector $v_{\lambda}$. If $\mu$ is a weight space of $V_{\lambda}$, then $\left\{A_{q}^{\mu-\lambda} \cdot v_{\lambda}\right.$ | $\left.\lambda \gg \operatorname{sh}\left(T_{A_{q}^{\mu-\lambda}}\right), \mu \gg \operatorname{co}\left(T_{A_{q}^{\mu-\lambda}}\right)\right\}$ is a basis for $V_{\lambda}^{\mu}$. In particular, if $V_{\lambda^{\prime}}$ is an irreducible representation of $\mathrm{SU}\left(n^{\prime}\right)$ with weight space $\mu^{\prime}$ such that $\lambda-\mu=\lambda^{\prime}-\mu^{\prime}$, then the sets of monomial lowering operators giving rise to the bases for $V_{\lambda}^{\mu}$ and $V_{\lambda^{\prime}}^{\mu^{\prime}}$ coincide if and only if $\lambda \gg \operatorname{sh}\left(T_{A_{q}^{\mu-\lambda}}\right)$ and $\mu \gg \operatorname{co}\left(T_{A_{q}^{\mu-\lambda}}\right)$ precisely when $\lambda^{\prime} \gg \operatorname{sh}\left(T_{A_{q}^{\mu-\lambda}}\right)$ and $\mu^{\prime} \gg \operatorname{co}\left(T_{A_{q}^{\mu-\lambda}}\right)$.

Proof This follows from Theorem 3 and Proposition 12.

Example 17. Consider $\lambda^{\prime}=(3,2,1,0)$ and $\mu^{\prime}=$ $(1,2,2,1)$. The difference $\mu^{\prime}-\lambda^{\prime}$ is the same as $\mu-\lambda$ from Example 9, but $V_{\lambda^{\prime}}^{\mu^{\prime}}$ is only 4-dimensional. The corresponding lowering operators are the first four operators from Table 2. The other two fail because $\mu^{\prime}-$ $\operatorname{co}\left(T_{A}\right)$ is not nonincreasing; they are $(0,1,0,0)$ and $(1,2,1,0)$ respectively. Similarly, for $\lambda^{\prime \prime}=(2,2,1,0)$ and $\mu^{\prime \prime}=(0,2,2,1), V_{\lambda^{\prime \prime}}^{\mu^{\prime \prime}}$ is one-dimensional, with basis coming from the first operator in Table 2 .

Corollary 18. Let $n$ be a positive integer such that $\lambda$ has length smaller than $n$ and $\mu$ has length at most $n$, and let $V_{\lambda}(n)$ be the corresponding irreducible representation of $\mathrm{SU}(n)$. Then the set of lowering operators giving the bases for $V_{\lambda}^{\mu}(n)$ is independent of $n$.

Corollary 19. Suppose that $\lambda \gg \operatorname{sh}\left(T_{A_{q}^{\mu-\lambda}}\right)$ and $\mu \gg$ $\operatorname{co}\left(T_{A_{q}^{\mu-\lambda}}\right)$ for all $q$. Then the set of lowering operators giving the basis for $V_{\lambda}^{\mu}$ is precisely the PBW basis for Low $w^{\mu-\lambda}$.

\subsection{Representations of $\mathrm{U}(n)$}

The analysis of the previous sections also applies to representations of the unitary group $\mathrm{U}(n)$. Here, the weight lattice is $\mathbf{Z} L_{1} \oplus \cdots \oplus \mathbf{Z} L_{n}$, with the dominant weights given by $\left\{\lambda_{1} L_{1}+\cdots+\lambda_{n} L_{n} \mid \lambda_{1} \geq \lambda_{2} \geq\right.$ $\left.\cdots \geq \lambda_{n}\right\}$. Again, the irreducible representations are in one-to-one correspondence with the dominant weights, and we let $W_{\lambda}$ denote the irreducible representation with highest weight $\lambda$. 
The unitary group $\mathrm{U}(n)$ is a quotient of $\mathrm{SU}(n) \times S^{1}$ via the multiplication homomorphism $\mathrm{SU}(n) \times S^{1} \stackrel{\phi}{\rightarrow}$ $\mathrm{U}_{n}$, so a representation of $\mathrm{U}(n)$ is the same as a representation of $\mathrm{SU}(n) \times S^{1}$ that is trivial on $\operatorname{Ker}(\phi)=$ $\left\{\left(\mathrm{e}^{2 \pi \mathrm{i} s / n} I, \mathrm{e}^{-2 \pi \mathrm{i} s / n}\right) \mid 0 \leq s \leq n-1\right\}$. It can be shown that the irreducible representations of $\mathrm{U}(n)$ correspond to the irreducible representations of $\mathrm{SU}(n) \times S^{1}$ of the form $V_{\lambda^{\prime}} \otimes L^{\otimes r}$, where $r=\sum \lambda_{i}^{\prime}+k n$ for some $k \in \mathbf{Z}$; here, $L$ is the natural one-dimensional representation of $S^{1}$ [34, p. 232-233]. It is easy to check that the weights of such a representation are given by $\mu+(k, \ldots, k)$, where $\mu$ is any weight of $V_{\lambda^{\prime}}$. It follows that given a dominant weight $\lambda=\left(\lambda_{1}, \ldots, \lambda_{n}\right), W_{\lambda}$ corresponds to $V_{\lambda^{\prime}} \otimes L^{\otimes r}$, with $\lambda^{\prime}=\left(\lambda_{1}-\lambda_{n}, \ldots, \lambda_{n-1}-\lambda_{n}, 0\right)$ and $k=\lambda_{n}$. It is now obvious that the lowering operators given in Theorem 3 for $V_{\lambda^{\prime}}$ provide a basis of weight vectors for $V_{l}$; indeed, the basis is the same, but with the weights shifted up by $\left(\lambda_{n}, \ldots, \lambda_{n}\right)$.

Theorem 16 and Corollaries 18 and 19 have obvious analogues for $\mathrm{U}(n)$. We will not state them explicitly; they are obtained by combining the results from Section 3.3 with the following proposition.

Proposition 20. Let $\lambda$ and $\lambda^{\prime}$ be dominant weights for $\mathrm{U}(n)$. If there exists an integer $k$ such that $\lambda_{i}-\lambda_{i}^{\prime}=$ $\mu_{i}-\mu_{i}^{\prime}=k$ for $1 \leq i \leq n$, then the sets of monomial lowering operators giving the bases for $W_{\lambda}^{\mu}$ and $W_{\lambda^{\prime}}^{\mu^{\prime}}$ coincide.

Proof In both cases, the lowering operators come from the semistandard tableau with shape $\hat{\lambda}=\lambda-$ $\left(\lambda_{n}, \ldots, \lambda_{n}\right)=\lambda^{\prime}-\left(\lambda_{n}^{\prime}, \ldots, \lambda_{n}^{\prime}\right)$ and content $\hat{\mu}=$ $\mu-\left(\lambda_{n}, \ldots, \lambda_{n}\right)=\mu^{\prime}-\left(\lambda_{n}^{\prime}, \ldots, \lambda_{n}^{\prime}\right)$.

\section{Proof of the main theorem}

We prove Theorem 3 by showing that the basis of lowering operators corresponds to a known basis for the Weyl module construction of irreducible representations of $\mathfrak{s l}_{n}(\mathbf{C})$. This approach was introduced in [14] and expounded in [15]. Denote the classical representation of $\mathfrak{s l}_{n}(\mathbf{C})$ with underlying vector space $\mathbf{C}^{n}$ by $V$. Let $e_{1}, \cdots, e_{n}$ be the ordered basis which gives the standard coordinates of $\mathbf{C}^{n}$. Every irreducible representation of $\mathfrak{s l}_{n}(\mathbf{C})$ is a subrepresentation of tensor products of the fundamental representation, $V[15,34]$. Suppose $\lambda$ is a dominant weight normalized so that $\lambda_{n}=0$. Let $\sum \lambda_{i}=d$ so that $\lambda$ is both a partition of $d$ and a Young diagram with $d$ boxes and $k \leq n-1$ rows. Number the boxes of $\lambda$ from 1 to $d$ starting left to right and top to bottom. The permutation group $S_{d}$ acts on elements of $\otimes_{i=1}^{d} V$ by permuting the factors and also on the boxes of $\lambda$. We will write the permutation group action on the right.

Let $R$ denote the subgroup of permutations of $S_{d}$ that preserve the rows of $\lambda$, and let $C$ denote the subgroup of permutations that preserve the columns of $\lambda$. Recall that the Young symmetrizer for $\lambda$ is the element of the group algebra $\mathbf{C}\left[S_{d}\right]$ given by $c_{\lambda}=$ $\sum_{\sigma=r c, r \in R, c \in C}(-1)^{c} \sigma$ where $(-1)^{c}$ is the sign of the permutation $c$. Young symmetrizers figure prominently in the representation theory of $S_{d}$; indeed, each irreducible representation of $S_{d}$ is uniquely determined by a Young symmetrizer. According to Weyl and Schur,

$$
\begin{array}{r}
V_{\lambda}=\operatorname{span}\left\{\sum_{\sigma=r c, r \in R, c \in C}(-1)^{\operatorname{sign} c} v_{(1) \sigma} \otimes \cdots\right. \\
\left.\otimes v_{(d) \sigma} \mid v_{1}, \cdots, v_{d} \in\left\{e_{1}, \cdots, e_{n}\right\}\right\}
\end{array}
$$

or equivalently,

$$
V_{\lambda}=\otimes_{i=1}^{d} V \cdot c_{\lambda}
$$

as explained in [34].

Given a (not necessarily semistandard) tableau

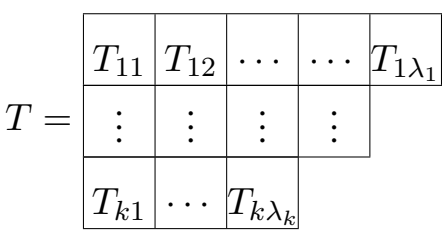

with $T_{i j} \in\{1, \cdots, n\}$, we let $v_{T}=e_{T_{11}} \otimes e_{T_{12}} \otimes$ $\cdots \otimes e_{T_{1 \lambda_{1}}} \otimes \cdots \otimes e_{T_{k 1}} \otimes \cdots \otimes e_{T_{k \lambda_{k}}} \cdot c_{\lambda}$ denote the corresponding vector in $V_{\lambda}$. It is easy to check that if the tableau $T$ has content $\mu$, then $v_{T} \in V_{\lambda}^{\mu}$. Note that as $T$ runs over these tableaux, the vectors

$w_{T}=e_{T_{11}} \otimes e_{T_{12}} \otimes \cdots \otimes e_{T_{1 \lambda_{1}}} \otimes \cdots \otimes e_{T_{k 1}} \otimes \cdots \otimes e_{T_{k \lambda_{k}}}$ give the standard basis for $\otimes_{i=1}^{d} V$, and $v_{T}$ may be written in terms of them via

$$
v_{T}=\sum_{\sigma=r c, r \in R, c \in C}(-1)^{c} w_{T \cdot \sigma} .
$$

Proposition 21. In the above notation, $V_{\lambda}^{\mu}$ has $\left\{v_{T} \mid\right.$ $\left.T \in \mathcal{S}_{\lambda}^{\mu}\right\}$ as a basis, i. e., those $v_{T} \mathrm{~s}$ corresponding to the semistandard tableaux on $\lambda$ with content $\mu$.

Proof This is a well-known result. However, the proofs we found in the literature showed the analogous fact for the vectors $v_{T}^{\prime}=\sum_{\sigma=c r, r \in R, c \in C}(-1)^{c} w_{T \cdot \sigma}$, 
where the order of column and row permutations is reversed. (See, for example, [34, Exercises 6.14, 6.15] or [47].) Accordingly, we give a sketch of the proof.

Since the dimension of $V_{\lambda}^{\mu}$ is equal to the size of $\mathcal{S}_{\lambda}^{\mu}[34]$, it suffices to show that the set $\left\{v_{T} \mid T \in \mathcal{S}_{\lambda}^{\mu}\right\}$ is linearly independent. To show independence, we make use of the notions of column equivalence and column dominance as described, for example, in [48]. Two tableaux with shape $\lambda$ are column equivalent if the content of each column of the two tableaux is the same. Hence, $T$ and $S$ are in the same equivalence class if and only if $S=T \cdot c$. There is a partial order $\unrhd$ on column equivalence classes of tableaux called column dominance. More formally, we will view $\unrhd$ as a preorder on the set of tableaux of a given shape with $T \unrhd S$ and $S \unrhd T$ implying that $T$ and $S$ are column equivalent. We leave it to the interested reader to seek the definition in [48], but we use the fact that it is a partial order on classes of tableaux of shape $\lambda$ and that $T \unrhd T \cdot r$ [48, Corollary 2.10.3]. Therefore $T \unrhd T \cdot(r c)$. Suppose $\sum_{T \text { semistandard }} a_{T} v_{T}$ is a linear combination, and let $T^{\prime}$ be a maximal semistandard tableau with $a_{T^{\prime}} \neq 0$. This means that if $T^{\prime}=T \cdot \sigma$ for any $T$ semistandard with $a_{T} \neq 0$, then $T=T^{\prime}$. Moreover, $T^{\prime}=T^{\prime} \cdot r c$ implies that $T^{\prime}=T^{\prime} \cdot r$ (or else $T^{\prime} \cdot r c$ is strictly smaller than $T^{\prime}$ ) and hence $c=1$ (since $T^{\prime}$ is strictly increasing down columns). Thus, if we let $k=\left|\left\{r \mid T^{\prime} \cdot r=T^{\prime}\right\}\right| \geq 1$, then

$$
\begin{aligned}
\sum_{T \text { semistandard }} a_{T} v_{T} & =\sum_{T \text { semistandard }} a_{T} \sum_{r c}(-1)^{c} w_{T \cdot r c} \\
& =\sum_{T \text { arbitrary }} b_{T} w_{T}
\end{aligned}
$$

with $b_{T^{\prime}}=k a_{T^{\prime}}$. Therefore, $\sum_{T \text { semistandard }} a_{T} v_{T} \neq 0$, and $\left\{v_{T} \mid T \in \mathcal{S}_{\lambda}^{\mu}\right\}$ is linearly independent set. This completes the proof.

Given a diagram $\lambda$, note that $\lambda$ is an admissible content, and there is a unique semistandard tableau with this content $\lambda$, namely, the tableau $L$ with all entries in the $i$ th row being $i$ :

$$
L=\begin{array}{c|c|c|c|c|}
\hline 1 & 1 & \ldots & \ldots & 1 \\
\hline \vdots & \vdots & \vdots & \vdots & \\
\cline { 1 - 2 } k & \cdots & k & \multicolumn{2}{|c}{} \\
\hline
\end{array}
$$

It is evident that a highest weight vector for $V_{\lambda}$ is given by $v_{\lambda}=v_{L}$. We will show that, for any semistandard tableau of shape $\lambda$,

$$
\gamma_{n \lambda}(T) \cdot v_{\lambda}=N_{T} \cdot v_{T}
$$

where $N_{T}$ is a positive integer. Combining this equation with Proposition 21 will complete the proof of Theorem 3 .

Remark 22. It follows from the argument below that for a semistandard tableau, $N_{T}$ is easily read from $\gamma_{n \lambda}(T)$ as the product of the factorials of the powers, e. g., for $T=\frac{2|2| 2|2| 3 \mid 3}{3|3| 3}, \gamma_{n \lambda}(T)=E_{21}^{4} E_{31}^{2} E_{32}^{3}$, and $N_{T}=4 ! 2 ! 3 !$. Notice that this multiplicity appears in the $\Sigma^{-}$row in Table 1. These multiplicities also appear in Carter and Lusztig, and the basis vectors that they give for $V_{\lambda}^{\mu}$ are in fact $\left(N_{T}\right)^{-1} \gamma_{n \lambda}(T) \cdot v_{\lambda}[44]$.

To demonstrate Equation (6), we need the following lemma.

Lemma 23. Suppose that $S$ is a tableau, $i \in\{1, \cdots, k\}$, and the following hypotheses are satisfied:

a. The only boxes with entry $i$ occur in the $i$ th row.

b. $S_{i j}=i$ for $1 \leq j \leq g$, where $g>0$, and $S_{i j}>i$ otherwise.

If $j_{1}>i$, then

1. $E_{j_{1} i} v_{S}=g v_{\hat{S}}$, where $S_{s t}=\hat{S}_{s t}$ for all $(s, t)$ except $(s, t)=(i, g)$ and $\hat{S}_{i g}=j_{1}$.

2. If $g>1$, then $\hat{S}$ also satisfies hypotheses (a) and (b).

Proof The proof is a straight calculation using the action of an operator on a tensor product. Note that

$$
\begin{aligned}
& v_{S}=e_{S_{11}} \otimes \cdots \otimes e_{S_{1 \lambda_{1}}} \otimes \cdots \otimes e_{S_{i 1}} \otimes \cdots \otimes e_{S_{i g}} \\
& \otimes e_{S_{i g+1}} \otimes \cdots \otimes e_{S_{i \lambda_{i}}} \otimes \cdots \otimes e_{S_{k 1}} \otimes \cdots \otimes e_{S_{k \lambda_{k}}} \cdot c_{\lambda} \\
& =e_{S_{11}} \otimes \cdots \otimes e_{S_{1 \lambda_{1}}} \otimes \cdots \otimes e_{i} \otimes \cdots \otimes e_{i} \otimes e_{S_{i g+1}} \\
& \otimes \cdots \otimes e_{S_{i \lambda_{i}}} \otimes \cdots \otimes e_{S_{k 1}} \otimes \cdots \otimes e_{S_{k \lambda_{k}}} \cdot c_{\lambda},
\end{aligned}
$$


with the $e_{i}$ s occurring exactly as $e_{S_{i 1}}, \cdots, e_{S_{i g}}$. Then,

$$
\begin{aligned}
E_{j_{1} i} v_{S} & =e_{S_{11}} \otimes \cdots \otimes e_{S_{1 \lambda_{1}}} \otimes \cdots \otimes E_{j_{1} i} e_{i} \otimes \cdots \\
& \otimes e_{i} \otimes e_{S_{i g+1}} \otimes \cdots \otimes e_{S_{i \lambda_{i}}} \otimes \cdots \otimes e_{S_{k 1}} \otimes \cdots \\
& \otimes e_{S_{k \lambda_{k}}} \cdot c_{\lambda}+\cdots \\
& +e_{S_{11}} \otimes \cdots \otimes e_{S_{1 \lambda_{1}}} \otimes \cdots \otimes e_{i} \otimes \cdots \otimes E_{j_{1} i} e_{i} \\
& \otimes e_{S_{i g+1}} \otimes \cdots \otimes e_{S_{i \lambda_{i}}} \otimes \cdots \otimes e_{S_{k 1}} \otimes \cdots \\
& \otimes e_{S_{k \lambda_{k}}} \cdot c_{\lambda} \\
& =g \cdot e_{S_{11}} \otimes \cdots \otimes e_{S_{1 \lambda_{1}}} \otimes \cdots \otimes e_{i} \otimes \cdots \\
& \otimes e_{i} \otimes e_{j_{1}} \otimes e_{S_{i g+1}} \otimes \cdots \otimes e_{S_{i \lambda_{i}}} \otimes \cdots \\
& \otimes e_{S_{k 1}} \otimes \cdots \otimes e_{S_{k \lambda_{k}}} \cdot c_{\lambda} \\
& =g v_{\hat{S}}
\end{aligned}
$$

since entries in the $S_{i 1}, \cdots, S_{i g}$ positions may be interchanged via a permutation that leaves the rows invariant.

Proof [Proof of Equation (6)]. We apply Lemma 23 repeatedly. Suppose $T$ is a semistandard tableau with shape $\lambda$. We set the following notation: $T_{i, 1}=\cdots=$ $T_{i, s_{i}}=i$, so the first entry bigger than $i$ in the $i$ th row is in column $s_{i}+1$; of course, $s_{i}$ may be zero. The tableaux that play the role of $S$ in applications of Lemma 23 are the tableau $L$ from (5) above and the tableaux $T(i)$ defined below, not $T$ itself. We examine the effect of

$$
\begin{aligned}
& \gamma_{n \lambda}(T)=\left(E_{T_{1 s_{1}+1} 1} \cdots E_{T_{1 \lambda_{1}} 1}\right) \cdots \\
& \left(E_{T_{i_{i}+1} i} \cdots E_{T_{i \lambda_{i}} i}\right) \cdots\left(E_{T_{k s_{k}+1} k} \cdots E_{T_{k \lambda_{k}} k}\right)
\end{aligned}
$$

on $v_{\lambda}=v_{L}$. The operators corresponding to a single row are blocked in parentheses. We first observe that

$$
\left(E_{T_{k s_{k}+1} k} \cdots E_{T_{k \lambda_{k}} k}\right) \cdot v_{\lambda}=m_{k} v_{T(k)}
$$

for some positive integer $m_{k}$, where the tableau $T(k)$ matches $L$ in the first $k-1$ rows but matches $T$ in the $k$ th row. To see this fact, apply the $\lambda_{k}-s_{k}$ operators one at a time using Lemma 23 each time.

The initial tableau $L$ satisfies the hypotheses of the lemma. Applying the operators replaces the entries in the $k$ th row one at a time, from right to left, by the $k$ th row of $T$. Each step reduces the number of $k \mathrm{~s}$ in this row, since there are $\lambda_{k} k \mathrm{~s}$ in $L$ and only $\lambda_{k}-s_{k}$ operators, the hypotheses of Lemma 23 hold to allow the next application. One ends up with a positive integer multiple of $v_{T(k)}$.

For $j=0, \cdots, k-1$, let $T(j+1)$ be the tableau of shape $\lambda$ that matches $L$ in the first $j$ rows and matches $T$ in the rows below $j$. The entries in rows $j+1$ to $k$ are all strictly greater than $j$ and $T(j+1)$ satisfies the hypotheses of Lemma 23. Now repeat the argument moving up the rows. Observe that

$$
\left(E_{T_{i s_{i}+1} i} \cdots E_{T_{i \lambda_{i}} i}\right) \cdot v_{T_{i+1}}=m_{i} v_{T(i)},
$$

as follows. There are $\lambda_{i}$ entries in the $i$ th row of $L$ that are $i$, and there are $\lambda_{i}-s_{i}$ operators. We can apply Lemma 23 repeatedly; the hypotheses hold at each step due to its Conclusion (2). The entries in the $i$ th row are replaced one at a time, from right to left, by the $i$ th row of $T$.

Since $T(1)=T$, Equation (6) is established.

By Proposition 21 and Eq. $(6),\left\{\gamma_{\lambda}^{\mu}(T) \cdot v_{\lambda} \mid T \in \mathcal{S}_{\lambda}^{\mu}\right\}$ is a basis for $V_{\lambda}^{\mu}$, thus demonstrating Theorem $\beta$.

Unlike the situation for crystal bases, those lowering operators in the PBW basis for $\mathcal{U}(\overline{\mathfrak{n}})$ not coming from $\mathcal{S}_{\lambda}(n)$ do not necessarily kill $v_{\lambda}$. However, if $A$ is a basis operator which does not fit inside $\lambda$ in the sense of Remark 15, then $A \cdot v_{\lambda}=0$.

Proposition 24. Let $A=\prod_{j<i \leq n} E_{i j}^{a_{i j}}$ be a monomial lowering operator (with the product in our usual order). If $A$ cannot be represented as $\gamma(T)$ for $T$ a (not necessarily semistandard) tableau of shape $\lambda$, then $A \cdot v_{\lambda}=0$.

Proof The hypothesis is equivalent to the statement that $\sum_{i>j} a_{i j}>\lambda_{j}$ for some $j$; let $s$ be the largest such index. Let $S$ be the tableau of shape $\lambda$ with nondecreasing rows which agrees with the highest weight tableau $L$ for the first $s$ rows while, for $j>s$, the $j$ th row contains $a_{i j} i$ s and $\lambda_{j}-\sum_{i>j} a_{i j} j \mathrm{~s}$. The same argument as in the proof above shows that $\prod_{s<j<i \leq n} E_{i j}^{a_{i j}} \cdot v_{L}=q v_{S}$ for some constant $q$. Applying the rightmost $\lambda_{s}$ operators of the form $E_{i s}$ to $v_{S}$ gives a sum of tensor products of the standard basis elements of $V$, none of which contain $e_{s}$. This sum will be killed by the next $E_{i s}$.

\section{Acknowledgements}

We would like to thank Martin Sage and Kameshwar Wali for helpful discussions and suggestions. We would 
also like to thank the referee for his useful comments. The research of the first author was partially supported by NSF grant DMS-0606300 and NSA grant H9823009-1-0059.

\section{References}

[1] L.C. Biedenharn and J.D. Louck, Angular Momentum in Quantum Physics (Addison-Wesley, Reading, 1981).

[2] A. Arima, Elliott's SU(3) model and its developments in nuclear physics, J. Phys. G 25, 581-588 (1999).

[3] J.P. Elliott, Collective motion in the nuclear shell model I. Classification schemes for states of mixed configurations, Proc. R. Soc. London Ser. A 245, 128-145 (1958).

[4] J.P. Elliott, Collective motion in the nuclear shell model II. The introduction of intrinsic wave-functions, Proc. R. Soc. London Ser. A 245, 562-581 (1958).

[5] H. Georgi, Lie Algebras in Particle Physics, 2nd ed. (Perseus Books, Reading, 1999).

[6] M. Moshinsky, Group Theory and the Many-Body Problem (Gordon and Breach, New York, 1968).

[7] J. Paldus, Unitary group approach to many-electron correlation problem, in: The Unitary Group, Lecture Notes in Chemistry, Vol. 22 (Springer-Verlag, New York, 1981) pp. 1-50.

[8] The Unitary Group, ed. J. Hinze, Lecture Notes in Chemistry , Vol. 22 (Springer-Verlag, New York, 1981).

[9] F.A. Matsen and R. Pauncz, The Unitary Group in Quantum Chemistry (Elsevier, Amsterdam, 1986).

[10] I. Bengtsson and K. Zyczkowski, Geometry of Quantum States: An Introduction to Quantum Entanglement (Cambridge University Press, Cambridge, 2008).

[11] D. Uskov and A.R.P. Rau, Geometric phases and Bloch-sphere constructions for $\mathrm{SU}(N)$ groups with a complete description of the SU(4) group, Phys. Rev. A 78, 022331 (2008).

[12] M. Moshinsky, Bases for the irreducible representations of the unitary groups and some applications, J. Math. Phys. 4, 1128-1139 (1963).10.1002/qua.560410105

[13] F.A. Matsen, Scientific reminiscences, Int. J. Quant. Chem. 41, 7-14 (1992).

[14] I. Schur, Über die rationalen Darstellungen der allgemeinen linearen Gruppe, 1927, in: I. Schur, Gesammelte Abhandlungen III (Springer, Berlin, 1973) pp. 68-85.

[15] H. Weyl, The Classical Groups (Princeton University Press, Princeton, NJ, 1997).

[16] H. Weyl, The Theory of Groups and Quantum Mechanics (Dutton, New York, 1931).

[17] J.G. Nagel and M. Moshinsky, Operators that lower or raise the irreducible vector spaces of $\mathrm{U}_{n-1}$ contained in an irreducible vector space of $\mathrm{U}_{n}$, J. Math. Phys. 6,
682-694 (1965).

[18] R.M. Asherova, Yu.F. Smirnov, and V.N. Tolstoi, Projection operators for simple Lie groups, Teoret. Mat. Fiz. 8, 255-271 (1971) [in Russian; English translation: Theoret. Math. Phys. 8, 813-825 (1971)].

[19] R.M. Asherova, Yu.F. Smirnov, and V.N. Tolstoi, Projection operators for simple Lie groups II. General scheme for constructing lowering operators. The groups $S U(n)$, Teoret. Mat. Fiz. 15, 107-119 (1973) in Russian; English translation: Theoret. Math. Phys. 15, 392-401 (1973)].

[20] D.P. Zhelobenko, Extremal projectors and generalized Mickelsson algebras on reductive Lie algebras, Izv. Akad. Nauk SSSR Ser. Mat. 52, 758-773, 895 (1988) in Russian; English translation: Math. USSR Izv. 33, 85-100 (1989).

[21] S. Khoroshkin and O. Ogievetsky, Mickelsson algebras and Zhelobenko operators, J. Algebra 319, 2113-2165 (2008).

[22] J. Mickelsson, Step algebras of semi-simple subalgebras of Lie algebras, Rep. Math. Phys. 4, 307-318 (1973).

[23] D.P. Zhelobenko, S-algebras and Verma modules over reductive Lie algebras, Soviet Math. Dokl. 28, 696-700 (1983).

[24] A.I. Molev, Gelfand-Tsetlin bases for classical Lie algebras, in: Handbook of Algebra, Vol. 4, ed. M. Hazewinkel (Elsevier, 2006) pp. 109-170.

[25] A.I. Molev, Weight bases of Gelfand-Tsetlin type for representations of classical Lie algebras, J. Phys. A 33, 4143-4168 (2000).

[26] V.N. Tolstoy, Extremal projectors for quantized KacMoody superalgebras and some of their applications, in: Quantum Groups, eds. H.D. Doebner and J.D. Hennig, Lecture Notes in Physics, Vol. 370 (Springer, Berlin, 1990) pp. 118-125.

[27] V.N. Tolstoy, Projection operator method for quantum groups, in: Special Functions 2000, NATO Science Series II, Vol. 30 (Kluwer Acad. Publ., The Netherlands, 2001) pp. 457-488.

[28] A.I. Molev, Gelfand-Tsetlin basis for Yangians, Lett. Math. Phys. 30, 53-60 (1994).

[29] A. Klimyk and K. Schmüdgen, Quantum Groups and Their Representations, Texts and Monographs in Physics (Springer-Verlag, Berlin, 1997).

[30] K. Ueno, T. Takebayashi, and Y. Shibukawa, Construction of Gel'fand-Zetlin basis for $U_{q}(q l(N+1))$ modules, Publ. RIMS Kyoto Univ. 26, 667-679 (1990).

[31] V.N. Tolstoy, Fortieth anniversary of extremal projector method for Lie symmetries, in: Noncommutative Geometry and Representation Theory in Mathematical Physics, eds. J. Fuchs, J. Mickelsson, G. Rozenblioum, A. Stolin, and A. Westerberg, Contemporary Mathematics, Vol. 391 (American Mathematical Society, 2005) p. 371-384 (2005). 
[32] M.E. Taylor, Noncommutative Harmonic Analysis (American Mathematical Society, Providence, 1986).

[33] D.P. Zelobenko, Compact Lie Groups and Their Representations (American Mathematical Society, Providence, 1973).

[34] W. Fulton and J. Harris, Representation Theory: A First Course (Springer-Verlag, New York, 1991).

[35] M. Kashiwara, On crystal bases of the $q$-analogue of universal enveloping algebras, Duke Math. J. 63, 465516 (1991).

[36] G. Lusztig, Canonical bases arising from quantized enveloping algebras, J. Am. Math. Soc. 2, 447-498 (1990).

[37] J. Hong and H. Lee, Young tableaux and crystal $B(\infty)$ for finite simple Lie algebras, J. Algebra 320, 36803693 (2008).

[38] B. Leclerc and P. Toffin, A simple algorithm for computing the global crystal basis of an irreducible $\mathrm{U}_{n}\left(\mathfrak{s l}_{n}\right)$-module, Int. J. Algebra Comput. 10, 191-208 (2000)

[39] H. Lee, Nakajima monomials and crystals for special linear algebras, Nagoya Math. J. 188, 31-57 (2007).

[40] J. Paldus and C.R. Sarma, Clifford algebra unitary group approach to many-electron correlation problem, J. Chem. Phys. 83, 5135-5152 (1985).

[41] F.A. Matsen, Spin-free quantum chemistry. XXIII. The generator-state approach, Int. J. Quantum Chem. 32, 71-86 (1987).

[42] D.J. Rowe, Properties of overcomplete and nonorthogonal basis vectors, J. Math. Phys. 10, 1774-1777 (1969).

[43] P.O. Löwdin, Quantum theory of cohesive properties of solids, Adv. Phys. 5, 1-172 (1956).

[44] R.W. Carter and G. Lusztig, On the modular representations of the general linear and symmetric groups, Math. Z. 136, 193-242 (1974).

[45] J.E. Humphreys, Introduction to Lie Algebras and Representation Theory (Springer-Verlag, New York, 1972.

[46] F.A. Matsen, Canonical generator states and their symmetry adaptation, Int. J. Quant. Chem. 18, 43-56 (1984).

[47] K. Akin, D. Buchsbaum, and J. Weyman, Schur functors and Schur complexes, Adv. Math. 44, 207-278 (1982).

[48] B.E. Sagan, The Symmetric Group, 2nd ed. (SpringerVerlag, New York, 2001).

\title{
ŽEMINANČIŲJŲ OPERATORIŲ, SKIRTŲ UNITARINIŲ GRUPIŲ NEREDUKUOTINIAMS ATVAIZDAMS, IŠREIKŠTINE் BAŻ
}

\author{
D.S. Sage, L. Smolinsky \\ Luizianos valstijos universitetas, Baton Ružas, JAV
}

\begin{abstract}
Santrauka
Unitariniu grupių atvaizdų teorija fundamentaliai svarbi daugelyje fizikos ir chemijos sričių. Unitarinès simetrijos fizikinès sistemos būsenų žymèjimui reikia turèti išreikštines bazes neredukuotiniams atvaizdams. Vienas sisteminių būdų gauti bazes yra apibendrinti laiptinių operatorių metodą $\mathrm{SU}(2)$ atvaizdams, panaudojant žeminančiųjų operatorių formalizmą. Čia nustatoma bazè visų žeminančiųų operatorių algebrai ir kiekvienam neredukuotiniam atvaizdui pateikiama instrukcija, kaip parinkti žeminančiųjų operatorių rinkinio dalį, kurią naudojant bazè gaunama iš didžiausio svorio vektoriaus. Bazės, gautos žeminančiaisiais operatoriais, ypač pato-
\end{abstract}

gios skaičiuojant stebimų dydžių matricinius koeficientus, kadangi jie virsta komutacijos sąryšiais standartiniams matriciniams vienetams. Žinomiausi šito metodo pavyzdžiai yra Gelfando ir Cetlino bazès sukonstravimas naudojant kraštutinius projektorius bei kristalinès (arba kanoninès) Kašivaros ir Lustigo bazès. Straipsnyje aprašomas kitas paprastas būdas gauti neredukuotinių atvaizdų bazes naudojant žeminančiuosius operatorius. Šios bazės nepasižymi Gelfando ir Cetlino ar kristalinių bazių kanoniškumu, tačiau kombinatorika, su kuria susiduriama, yra daug paprastesnè ir dẻl to šios bazès ypač tinka fizikiniams taikymams. 